\title{
Erratum to: Mather Measures Associated with a Class of Bloch Wave Functions
}

Olga Bernardi, Alberto Parmeggiani and Lorenzo Zanelli

\section{Erratum to: Ann. Henri Poincaré 13 (2012), 1807-1839 DOI 10.1007/s00023-012-0174-z}

Regrettably, we erroneously wrote the norm $\|\cdot\|_{C^{0,1}}$ for the norm $\|\cdot\|_{C^{1}}$. The norm has to be corrected throughout the paper, as well as the reference to $C^{0,1}$-critical subsolutions that has to be replaced by $C^{1,1}$-critical subsolutions (whose existence is granted by a result of Bernard [1], reference [9] in the paper). In other words, in the paper the wave functions are constructed in terms of suitable $C^{k}$-phases which approximate $C^{1,1}$-critical subsolutions of the Hamilton-Jacobi equation. Hence:

- The norm has to be corrected on page 1810 l. 13; page 1824 formulas (4.22) and (4.25), and lines 2 and 5 below (4.25); page 1825 formula (4.26), (ii) in Def. 4.12, l. 1 below formula (4.27); page 1836 l. 2 below formula (5.14) and bottom line.

- A $C^{0,1}$-critical subsolution has to be replaced by a $C^{1,1}$-critical subsolution on page 1810 l. 5; page 1811 l. 1 below formula (1.13); page 1816 l. 1 of Prop. 3.1; page 1824 l. 2 above formula (4.22), in the formula (4.24) and (4.25); page 1825 formula (4.26), l. 3 of Def. 4.12 and 1.1 below formula (4.27); page 1836 formula (5.13), (5.14), 1. 2 and 1. 4 below (5.14), 1. 1 above (5.15), (5.15), (5.16) and 1. 2 below (5.16).

The online version of the original article can be found under doi:10.1007/s00023-012-0174-z. 
Unfortunately there are other imprecisions, that we now list:

- Page 1813 lines 8 and 11 below formula (2.4): $x$ has of course to be replaced by $x_{0}$.

- Page 1813 l. 11 of point 3: "weak KAM solutions of negative type solve the H-J equation in the viscosity sense".

- Page 1816 line 4 from bottom of page: $C^{0,1}$ has of course to be replaced by $C^{1}$ so as to read "the density of $C^{\infty}$ into $C^{1}$ ".

- Page 1816 line 3 from bottom of page: the convergence of $\nabla_{x} g_{k}(P, \cdot)$ to $\nabla_{x} v(P, \cdot)$ is in the $C^{0}$ topology.

- Page 1820, the correct statement of Thm. 4.5 is: For $0<\alpha<1$ arbitrarily fixed, for all $P \in \mathbb{R}^{n}$. we have the inequalities

$$
\bar{H}(P)-2 \hbar^{\alpha} \leq \bar{H}_{\hbar}(P) \leq \bar{H}(P)
$$

for all $0 \leq \hbar \leq 1$.

- Page 1822 1. 3 below formula (4.18): " $\forall 0<\alpha<1$ " has to be replaced by " $0<\alpha<1$ ".

- Page 1824 lines 1 and 2 below formula (4.22): "This fact is always possible thanks to the density of $C^{k}\left(\mathbb{T}^{n} ; \mathbb{R}\right)$ in $C^{1}\left(\mathbb{T}^{n} ; \mathbb{R}\right)$ ".

- Page 1825 line 4 of Def. 4.12: " $\forall 0<\alpha<1$ " has to be replaced by "for some $0<\alpha<1$ ".

- Page 1825 line 1 of Prop. 4.15: "and all $0<\alpha<1$ " has to be deleted.

- Page 1828 Lemma 5.4: conclusion (a) of the lemma should be stated more precisely as

$$
\begin{gathered}
\varepsilon^{n} \sum_{\alpha \in \varepsilon \mathbb{Z}^{n}} f(\alpha) e^{i \alpha \cdot y}=(2 \pi)^{n}\left(\mathcal{F}^{-1} f\right)(y)+F(\varepsilon, y), \forall y \in \varepsilon^{-1} Q_{n} \\
\forall N \in \mathbb{Z}_{+} \exists C_{f, N} \text { s.t. }\|F(\varepsilon, \cdot)\|_{L^{\infty}\left(\varepsilon^{-1} Q_{n}\right)} \leq C_{f, N} \varepsilon^{N} .
\end{gathered}
$$

- Page 1828 l. 1 above formula (5.4): "the error term $F(\varepsilon, y)$, for $y \in \varepsilon^{-1} Q_{n}$, is given by ..."

- Page 1832 line 5 from bottom of page: $\operatorname{vol}(\operatorname{supp} F(\hbar, \cdot))$ has to be replaced by $\operatorname{vol}\left(D_{\hbar, n}\right)$; the norm is $\|F(\hbar, \cdot)\|_{L^{\infty}\left(D_{\hbar, n}\right)}$.

- Page 1834 line 6 from bottom of page: the norm is $\|F(\hbar, \cdot)\|_{L^{\infty}\left(D_{\hbar, n}\right)}$.

- Page 1835 lines 5 and 6 of the proof of Thm. 1.2: the $C^{0,1}$-convergence has of course to be replaced by the $C^{1}$-convergence; recall that the critical subsolutions referred to are $C^{1,1}$-critical subsolutions; lines 10-12 of the same proof should be rephrased as: ". . . all have the same support, namely $\mathbb{T}^{n}$, and so they form in fact a tight family of probability measures (see [13]). Thus, an application of the Prohorov Theorem (see for example Thm. 5.1 of [13]) ensures there exists a weak* convergent sequence ..."

- Page 1836 line 7 below formula (5.14): of course, $C^{0,1}$ has to be replaced by $C^{1}$ so as to read "by using the density of $C^{\infty}\left(\mathbb{T}^{n} ; \mathbb{R}\right)$ in $C^{1}\left(\mathbb{T}^{n} ; \mathbb{R}\right)$ ".

- Page 1836 bottom line: $P$ has to be replaced by $|P|$. 


\section{Reference}

[1] Bernard, P.: Existence of $C^{1,1}$ critical sub-solutions of the Hamilton-Jacobi equation on compact manifolds. Ann. Sci. École Norm. Sup. (4) 40(3), 445-452 (2007)

Olga Bernardi

Department of Mathematics

University of Padova

Via Trieste, 63

35121 Padova, Italy

e-mail: obern@math.unipd.it

Alberto Parmeggiani and Lorenzo Zanelli

Department of Mathematics

University of Bologna

Piazza di Porta San Donato, 5

40126 Bologna, Italy

e-mail: alberto.parmeggiani@unibo.it;

lorenzo.zanelli@unibo.it 